\title{
Mechanisms for redox-regulation of protein kinase $\mathbf{C}$
}

\author{
Susan F. Steinberg* \\ Department of Pharmacology, College of Physicians and Surgeons, Columbia University, New York, NY, USA
}

Protein kinase $\mathrm{C}(\mathrm{PKC})$ is comprised of a family of signal-regulated enzymes that play pleiotropic roles in the control of many physiological and pathological responses. PKC isoforms are traditionally viewed as allosterically activated enzymes that are recruited to membranes by growth factor receptor-generated lipid cofactors. An inherent assumption of this conventional model of PKC isoform activation is that PKCs act exclusively at membrane-delimited substrates and that PKC catalytic activity is an inherent property of each enzyme that is not altered by the activation process. This traditional model of PKC activation does not adequately explain the many welldocumented actions of PKC enzymes in mitochondrial, nuclear, and cardiac sarcomeric

OPEN ACCESS

Edited by: Kristina Lorenz,

University of Wuerzburg, Germany

Reviewed by:

Wolfgang F. Graier,

Medical University of Graz, Austria Gaetano Santulli,

The University of Naples Federico II,

Italy

*Correspondence: Susan F. Steinberg

Department of Pharmacology, College of Physicians and Surgeons, Columbia University, 630 West 168 Street, New York, NY 10032, USA sfs1@columbia.edu

Specialty section:

This article was submitted to Cardiovascular and Smooth Muscle Pharmacology, a section of the journal Frontiers in Pharmacology

Received: 01 May 2015 Accepted: 10 June 2015 Published: 23 June 2015

Citation:

Steinberg SF (2015) Mechanisms for redox-regulation of protein

kinase $C$

Front. Pharmacol. 6:128. doi: 10.3389/fphar.2015.00128 (non-sarcolemmal) subcellular compartments. Recent studies address this dilemma by identifying stimulus-specific differences in the mechanisms for PKC isoform activation during growth factor activation versus oxidative stress. This review discusses a number of non-canonical redox-triggered mechanisms that can alter the catalytic properties and subcellular compartmentation patterns of PKC enzymes. While some redoxactivated mechanisms act at structural determinants that are common to all PKCs, the redox-dependent mechanism for $\mathrm{PKC} \delta$ activation requires Src-dependent tyrosine phosphorylation of a unique phosphorylation motif on this enzyme and is isoform specific. Since oxidative stress contributes to pathogenesis of a wide range of clinical disorders, these stimulus-specific differences in the controls and consequences of PKC activation have important implications for the design and evaluation of PKC-targeted therapeutics.

Keywords: protein kinase C, oxidative stress, tyrosine phosphorylation, Src, post-translational modifications

\section{Introduction}

Protein kinase $\mathrm{C}$ (PKC) comprises a multigene family of related serine/threonine kinases that mediate a vast number of cellular signaling responses. Cardiomyocytes co-express multiple PKC isoforms that have been implicated in responses as diverse as cardiac contraction, cardiac hypertrophy, cardiac fibrosis, ischemia/reperfusion injury, and ischemic preconditioning (Steinberg, 2008, 2012). PKC isoforms were initially characterized as lipid-sensitive enzymes that are activated by growth factor receptors that stimulate phospholipase C (PLC; Figure 1). PLC exists as a family of enzymes with highly conserved catalytic domains that hydrolyzes phosphatidylinositol 4,5-bisphosphate $\left(\mathrm{PIP}_{2}\right)$ to generate the calcium-mobilizing second messenger molecule inositol trisphosphate $\left(\mathrm{IP}_{3}\right)$ and diacylglycerol $(\mathrm{DAG})$, the lipid cofactor that allosterically activates PKC. PLC enzymes are subdivided into subclasses based upon the distinctive structural features in their regulatory domains and their diverse roles in cellular responses. PLC $\beta$ 


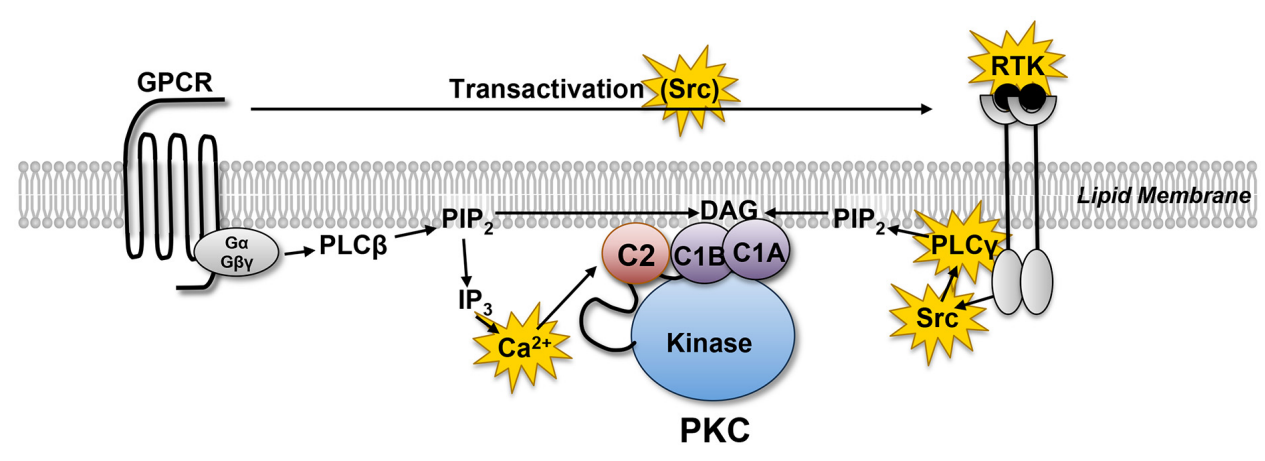

FIGURE 1 | Receptor-dependent mechanisms that allosterically activate protein kinase $\mathbf{C}$ (PKC). G protein coupled receptors (GPCRs) and receptor tyrosine kinases (RTKs) activate distinct phospholipase C (PLC) family enzymes. While the regulatory controls for different PLC family enzymes differ, these enzymes act in a similar manner to hydrolyze membrane $\mathrm{PIP}_{2}$ leading to the generation of the calcium-mobilizing second messenger $\mathrm{IP}_{3}$ and $\mathrm{DAG}$. The redox-sensitive elements in this pathway are highlighted in yellow (see text). isoforms are activated by heterotrimeric $G$ protein subunits (G $\alpha q$ and $G \beta \gamma$ ) and mediate G protein-coupled receptor (GPCR) responses. In contrast, PLC $\gamma$ isoforms are recruited to activated receptor tyrosine kinases (RTKs) where they are activated as a result of tyrosine phosphorylation by receptor or non-receptor tyrosine kinases. Since many GPCRs transactivate RTKs, PLC- $\gamma$ isoforms have been implicated in both RTK and GPCR signaling responses.

Conventional models of PKC activation focus exclusively on the second messenger-driven translocation mechanisms that deliver these enzymes in an active conformation to their membrane-delimited substrates. An inherent assumption of this model is that PKC isoforms act as generic enzymes with little-to-no substrate specificity. However, studies over the past several years have exposed additional mechanisms that alter the cofactor-requirements and/or catalytic properties of individual PKCs. Among these, the redox-dependent mechanisms that regulate $\mathrm{PKCs}$ are particularly interesting, since oxidative stress is a prominent feature of most clinically important cardiac disorders. While there is ample evidence that increased levels of reactive oxygen species (ROS) result in adverse cardiac remodeling and deleterious changes in contractile performance through direct redox-dependent modifications of calcium regulatory proteins and/or structural proteins in the sarcomere, this review focuses on redox-activated mechanisms that directly or indirectly influence signaling by individual PKCs. These constitute alternative mechanisms to influence pump function and/or the pathogenesis of heart failure that could provide strategies for the development of novel therapeutics.

\section{The Structural Features that Regulate Individual Isoforms of PKC}

Protein kinase $\mathrm{C}$ isoforms are structurally defined by a highly conserved C-terminal catalytic domain (consisting of motifs required for $\mathrm{ATP} /$ substrate-binding and catalysis) that is separated by a flexible hinge region from the $\mathrm{N}$-terminal regulatory domain (Figure 2). Structural features in the regulatory domain provide the basis to subdivide $\mathrm{PKCs}$ into distinct subfamilies. Conventional PKC isoforms (cPKCs; $\alpha$, the alternatively spliced $\beta \mathrm{I} / \beta \mathrm{II}$ isoforms, and $\gamma$ ) contain two discrete membrane-targeting modules, termed $\mathrm{C} 1$, and $\mathrm{C} 2$. The $\mathrm{C} 1$ domain is comprised of tandem cysteine-rich $\mathrm{C} 1 \mathrm{~A}$ and $\mathrm{C} 1 \mathrm{~B}$ sequences, with a signature $\mathrm{HX}_{12} \mathrm{CX}_{2} \mathrm{CX}_{13-14}$ $\mathrm{CX}_{2} \mathrm{CX}_{4} \mathrm{HX}_{2} \mathrm{CX}_{7} \mathrm{C}$ motif (in which $\mathrm{C}$ is cysteine, $\mathrm{H}$ is histidine, and $\mathrm{X}$ is any residue). The six cysteine and two histidine residues in each of the twin $\mathrm{C} 1$ domain structures coordinate zinc ions that stabilize the zinc finger structures. $\mathrm{C} 1$ domains contain the binding sites for DAG and tumor-promoting phorbol esters such as phorbol 12-myristate 13-acetate (PMA). The C1 domain lipid-binding sites are surrounded by a band of hydrophobic residues that form an extended hydrophobic surface that penetrates the lipid bilayer and provides a strong driving force to anchor the $\mathrm{C} 1$ domains to the DAG-containing membrane. However, membrane-docking interactions based upon $\mathrm{C} 1$ domain binding to $\mathrm{DAG}$ are not sufficient to stabilize full-length cPKCs at membranes. Rather, cPKC stably associate with membranes as a result of a second membrane-targeting interaction involving the $\mathrm{C} 2$ domain. cPKCs $\mathrm{C} 2$ domains of are $\sim 130$ residue eight-stranded anti-parallel $\beta$-sandwich structures with three inter-strand $\mathrm{Ca}^{2+}$. binding loops (CBLs) responsible for calcium-dependent anionic phospholipid binding.

Novel PKCs (nPKCs, $\delta, \theta \varepsilon$, and $\eta$ ) also have twin $\mathrm{C} 1$ domains and a $\mathrm{C} 2$ domain, although the ordering of $\mathrm{nPKC}$ isoform $\mathrm{C} 1$ and $\mathrm{C} 2$ domains, along the linear sequence of the protein, is switched relative to the order in CPKC (Figure 2). While $\mathrm{nPKC} \mathrm{C1}$ domains function as lipid-binding membranetargeting modules (much like their counterparts in cPKCs), nPKCs are maximally activated by agonists that promote DAG accumulation or by PMA, without a calcium requirement. This difference can be attributed to a specific sequence difference between $\mathrm{CPKC}$ and $\mathrm{nPKC} \mathrm{C} 2$ domain. nPKC C2 domains lack the critical calcium-coordinating aspartic acid residues that are highly conserved in cPKCs. As a result, the PKCE C2 domain does not bind calcium; it is a membrane-targeting 


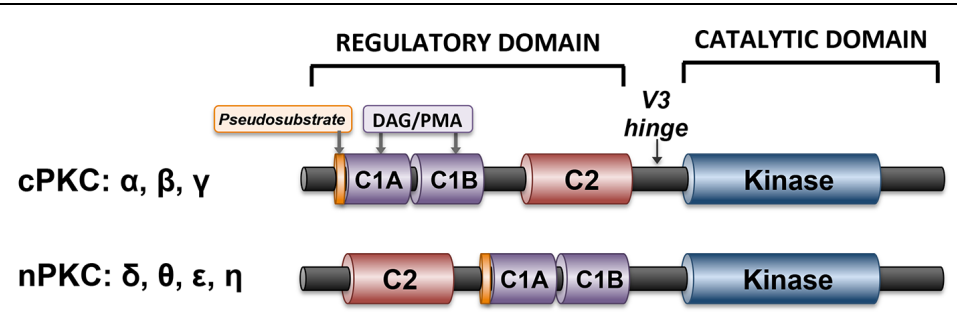

PKC ISOFORM-SPECIFIC DIFFERENCES IN C2 DOMAIN FUNCTION

cPKC: $\mathrm{Ca}^{2+}$-dependent anionic phospholipid binding motif

PKC $\varepsilon: \quad \mathrm{Ca}^{2+}$-independent membrane-targeting motif

PKCס: Protein-protein interaction motif

Interacts with $p Y$ flanked by the consensus

(Y/F)-(S/A)- (VII)-pY-(Q/R)-X-(Y/F) sequence

FIGURE 2 | Domain structure of cPKC and nPKC family enzymes. The conserved pseudosubstrate motif (shown in orange) N-terminal to the C1 domain (shown in lavender), the $\mathrm{C} 2$ domain (red), and the kinase domain (blue) and the more variable regions (shown in black) are depicted.

module that binds lipids in a calcium-independent manner. The PKC $\delta$ C2 domain is even more distinct from a functional standpoint, as it does not bind lipids. Rather it is a proteinprotein interaction domain that binds phospho-tyrosine residues flanked by the consensus sequence (Y/F)-(S/A)-(V/I)-pY-(Q/R)$\mathrm{X}-(\mathrm{Y} / \mathrm{F})$ (Benes et al., 2005). Recent studies indicate that the PKC $\theta$ C2 domain (which shares a high degree of structural homology to the $\mathrm{C} 2$ domain in $\mathrm{PKC} \delta$ ) also functions as a high affinity phosphotyrosine-binding motif (Stahelin et al., 2012). The general consensus is that PKC $\theta$ is a key signaling intermediate in immune cells and skeletal muscle, but that it not detected in cardiomyocytes (and it has at best only a minor role in cardiovascular responses).

Regulatory regions of both $\mathrm{cPKC}$ and $\mathrm{nPKC}$ also contain a pseudosubstrate motif just $\mathrm{N}$-terminal to their respective $\mathrm{C} 1$ domains. The pseudosubstrate motif sequence resembles a PKC substrate with an alanine substitution at the phosphoacceptor site (or P-site). For both cPKCs and nPKCs, an intramolecular autoinhibitory interaction between the regulatory domain peudosubstrate motif and the kinase domain substratebinding pocket maintains a low level of basal catalytic activity.

\section{Redox-Dependent Mechanisms that Generate Lipid Cofactors that Activate PKC}

Cells generate ROS through a variety of tightly controlled metabolic and cellular processes that derive from mitochondrial respiration and/or growth factor stimulation. High levels of ROS also are produced during ischemic injury or in response to many pathologic insults. There is growing evidence that the subcellular location or the chemical nature of the reactive species produced in a particular setting determine whether ROS function as second messengers in signal transduction pathways or as agents for cellular injury. A major mechanism for ROSinduced cellular regulation involves chemical modification of reactive thiols in cysteine (or to a lesser extent methionine) residues in proteins. The reactivity of a particular cysteine residue is determined by the $\mathrm{pK}_{\mathrm{a}}$ of its thiol moiety; cysteine residues adjacent to basic amino acids (Arg or Lys), aromatic amino acids, or metal centers, have relatively low $\mathrm{pK}_{\mathrm{a}}$ 's $(<6.5)$ and tend to be susceptible to oxidation. The resultant cysteine oxidation can lead to disulfide bond formation and conformational changes that alter protein structure and function. Since many enzymes have reactive cysteines in their catalytic centers, oxidation of these cysteine residues leads to enzyme inactivation. One important class of enzymes with extremely low $\mathrm{pKa}$ active-site catalytic cysteine residues is protein tyrosine phosphatases (PTPs). Since protein tyrosine phosphorylation is controlled through the reciprocal actions protein tyrosine kinases (PTKs) and PTPs - and PTPs are extremely sensitive to redox-inactivation - this tips the balance in favor of increased protein tyrosine phosphorylation. This explains PTPs strategic position as a mediator of oxidant stress-dependent signaling responses.

Oxidative stress and growth factor receptors activate many common pathways that culminate in the activation of PKCs. Two major factors explain the similarity between growth factor and redox-activated mechanisms. First, many membrane receptors couple to signaling pathways that culminate in changes in the expression and/or activity of ROS-generating or antioxidant enzymes. For example, GPCRs (such as angiotensin II, $\alpha_{1}$-adrenergic, or endothelin-1 receptors) activate signaling pathways that lead to increased ROS generation; the receptordependent increase in ROS in turn activates molecular targets that play critical roles in both physiologic and pathologic growth 
responses (Nakamura et al., 1998; Hirotani et al., 2002; Kuster et al., 2005; Guo et al., 2009).

Conversely, oxidative stress leads to the activation of certain components of the canonical GPCR- or RTK-driven signaling machinery that culminate in the activation of PKCs (Figure 1). For example, $\mathrm{H}_{2} \mathrm{O}_{2}$ treatment increases epithelial growth factor receptor (EFGR) phosphorylation and oxidation at $\mathrm{Cys}^{797}$, a critical residue in the active site of the kinase domain (Paulsen et al., 2012; Truong and Carroll, 2012). Recent studies establish that the direct $\mathrm{H}_{2} \mathrm{O}_{2}$-dependent oxidation of EGFRs at Cys ${ }^{797}$ increases tyrosine kinase activity (Paulsen et al., 2012). Redoxactivated EGFRs recruit PLC $\gamma$, which generates the lipid cofactor DAG that activates PKCs. Of note, two other EGFR family members (Her2 and Her4) and nine other receptor or nonreceptor PTKs in the human genome have cysteine residues at corresponding positions in their kinase domains, suggesting that cysteine oxidation might constitute a more common mechanism to activate a selected subset of other tyrosine kinases.

Phospholipase $\mathrm{C} \gamma$ also is a redox-sensitive protein. PLC $\gamma$ enzymes are activated when recruited via their Src homology (SH) 2 domains to phospho-tyrosine residues on activated (tyrosine-phosphorylated) RTKs. Receptor-anchored PLC $\gamma$ is then tyrosine phosphorylated at critical sites that are essential to increase PLC $\gamma$ activity. PLC- $\beta$ isoforms do not have SH2 domains, are not regulated through tyrosine phosphorylation, and are not redox-sensitive enzymes. A number of laboratories have demonstrated that oxidative stress leads to a functionally important increase in PLC $\gamma$ tyrosine phosphorylation (Wang et al., 2001). Studies of mechanism have variably attributed this to a direct redox-dependent increase in EGFR kinase activity (Wang et al., 2000), direct redox-dependent or indirect EGFR-dependent activation of Src kinases, and/or redox-dependent inhibition of PTPs. Importantly, the redox-activated PLC $\gamma$ enzyme activates a cytoprotective pathway that involves PKC (Wang et al., 2001).

Src-family non-receptor tyrosine kinases (which are critically regulated through stimulatory and inhibitory tyrosine phosphorylations) also are activated through a number of redoxdriven mechanisms. Src kinases are structurally characterized by regulatory $\mathrm{SH} 3$ and $\mathrm{SH} 2$ domains and a catalytic domain, that terminates in a C-terminal regulatory sequence containing an autoinhibitory tyrosine residue; Src kinases are maintained in a closed inactive conformation with low levels of basal enzyme activity as a result of an intramolecular autoinhibitory SH2 domain-C-terminal phospho-tyrosine interaction (Xu et al., 1999). Src kinases activation requires de-phosphorylation of the auto-inhibitory phospho-tyrosine ( $\mathrm{Tyr}^{527}$ in c-Src), which releases this autoinhibitory constraint; the enzyme then autophosphorylates at a positive regulatory site in the activation loop of the catalytic domain $\left(\mathrm{Tyr}^{418}\right.$ in $\mathrm{c}-\mathrm{Src}$ ). The $\mathrm{Src} \mathrm{SH} 2$ domain contains cysteine residues with $\mathrm{p} K_{\mathrm{a}}$ 's low enough to be oxidized by physiological levels of ROS. Direct oxidative modification of these $\mathrm{SH} 2$ domain cysteine residues leads to the formation of intra-molecular disulphide bridges that secondarily disrupt the autoinhibitory $\mathrm{Tyr}^{527}-\mathrm{SH} 2$ domain interaction, leading to enzyme activation (Knock and Ward, 2011; MacKay and Knock, 2014). Src also contains a cysteine residue at a strategic position in its glycine-rich ATP-positioning loop (G-loop, also known as the phosphate binding P-loop). This cysteine residue ( $\mathrm{Cys}^{277}$ in $\mathrm{Src}$ ) also has been implicated as a target for oxidative modifications that underlie redox-dependent changes in Src kinase activity (Zhang et al., 2015). Of note, two other Src family kinases (Yes and Fgr) and four members of the fibroblast growth factor receptor family have cysteine residues at corresponding positions in their $\mathrm{G}$ loops. This could suggest that oxidation of a cysteine residue strategically positioned in the enzyme catalytic center constitutes a mechanism to selectively regulate only a subset of PTKs. This is in marked contrast to PTPs, which are inactivated in a more general manner through direct oxidation of a common active site cysteine. Finally, direct oxidative modification (and inhibition) of the PTP that dephosphorylate the Src activation loop site also contribute to redox-activation of Src kinase activity (MacKay and Knock, 2014). Src kinases play an essential role in the redox-dependent mechanisms that activate $\operatorname{PKC} \delta$, which is discussed in the Section "Direct Redox Modifications of Cysteine Residues in PKCs."

There is limited evidence that free radicals can directly oxidize membrane phospholipids; PLC-dependent cleavage of phospholipid hydroperoxides results in the formation of diacylglycerol hydroperoxide which acts as a very potent stimulator of PKC in inflammatory neutrophils (Kambayashi et al., 2007). The relevance of oxidized lipid second messenger molecules at sites of inflammation to ischemiareperfusion injury in cardiovascular tissues has never been considered.

\section{Redox-Dependent Mechanisms Involving Calcium that Can Lead to the Activation of PKC}

Finally, it would be remiss to at least not mention the fact that excessive ROS can lead to calcium overload by altering the redox state of key calcium regulatory proteins. While a detailed discussion of this topic goes beyond the scope of this review [it is reviewed in Santos et al. (2011) and Tocchetti et al. (2015)], it is worth noting that several major proteins involved in excitation-contraction coupling are targets for functionally important oxidative modifications. Most studies have focused on redox modifications that target the major calcium regulatory proteins at the sarcoplasmic reticulum (SR), namely the ryanodine receptor (RyR) $\mathrm{Ca}^{2+}$-release channel or $\mathrm{SR} \mathrm{Ca}^{2+}$-ATPase (SERCA). RyRs are macromolecular complexes comprised of four RyR protein monomers (each with a molecular mass of $\sim 565 \mathrm{kDa}$ ) bound to channel stabilizing calstabin (or FK506 binding protein) subunits and various regulatory kinases, phosphatases, and phosphodiesterases (Zalk et al., 2007). Calstabin subunits maintain RyRs in a closed state that prevents pathologic $\mathrm{Ca}^{2+}$ leak. Any process that triggers RyR-calstabin dissociation enhances channel open probability and increases diastolic SR calcium leak (Umanskaya et al., 2014). Recent studies indicate that RyRs contain many cysteine residues (almost two dozen per monomer) that are highly 
susceptible to various redox modifications and that cysteinedirected oxidative modifications of RyRs disrupt the calstabinRyR interaction. Calstabin-dissociating redox modifications of RyRs (due to enhanced ROS generation by calcium-overloaded dysfunctional mitochondria) have been implicated in agerelated skeletal muscle dysfunction (Andersson et al., 2011), abnormalities in glucose-induced insulin secretion in pancreatic $\beta$ cells (Santulli et al., 2015), and disturbances in diastolic $\mathrm{Ca}^{2+}$ homeostasis leading to $\mathrm{Ca}^{2+}$ waves and ventricular extrasystoles in cardiomyocytes (Roussel et al., 2015). SERCA2a (the major SERCA isoform in cardiomyocytes) also is a target for oxidation at $\mathrm{Cys}^{674}$ and nitration at $\mathrm{Tyr}^{294} / \mathrm{Tyr}^{295}$; these oxidative modifications decrease maximal SERCA2 activity and lead to decreased calcium reuptake during diastolic relaxation. Finally, calcium overload can result from thiol oxidization of reactive cysteine residues in the pore-forming $\alpha 1 C$ subunit of the voltage-gated L-type $\mathrm{Ca}$ channel. This oxidative modification has been linked to increased channel activity.

Increased intracellular calcium can act as a cofactor to activate cPKCs through the conventional canonical pathway. However, increased calcium also would lead to the activation of calpain, a calcium-dependent cysteine protease that cleaves the $\mathrm{V} 3$ hinge region of $\mathrm{PKC} \alpha$. This proteolytic activation mechanism liberates a C-terminal constitutively active catalytic fragment (termed $\mathrm{PKM} \alpha$ ) that partitions to the nucleus and acts as an unregulated/mislocalized enzyme to stimulate gene programs that promote pathological structural and functional cardiac remodeling (Kang et al., 2010; Zhang et al., 2011). Of note, while PKC $\alpha$ is the most abundant PKC isoform in cardiomyocytes - and hence the cardiac actions of PKM $\alpha$ appear to predominate under conditions of calcium overload - calpain cleaves other cPKC isoforms and PKC $\delta$ (Kishimoto et al., 1989; Yamakawa et al., 2001). Hence, a calpain-dependent proteolytic activation mechanism may be a more general mechanism to activate other PKC isoforms; it may not be specific for $\mathrm{PKC} \alpha$.

\section{Direct Redox Modifications of Cysteine Residues in PKCs}

Both the $\mathrm{N}$-terminal regulatory region and the $\mathrm{C}$-terminal catalytic domains of PKCs contain cysteine residues that are susceptible to redox modifications (Figure 3). The cysteine residues in the regulatory $\mathrm{C} 1$ domain are particularly sensitive to oxidative modifications that disrupt this structure and lead to kinase activation. Studies of mechanism suggest that oxidative modification of these cysteine residues (which are precisely spaced to form a hairpin structure that coordinates zinc ions) result in the release of zinc atoms and a conformational change that disrupts the $\mathrm{C} 1$ domain lipid-binding pocket leading to the loss of phorbol ester binding activity (Knapp and Klann, 2000; Korichneva et al., 2002). However, the redox-activated enzyme (which does not bind phorbol esters) is nevertheless a catalytically active enzyme since the C1 domain-targeted oxidative modifications also disrupt the

\section{REDOX-SENSITIVE TARGETS COMMON TO ALL PKCS}

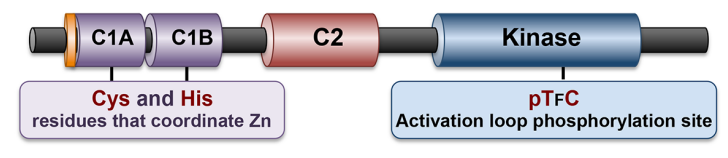

FIGURE 3 | Redox-sensitive residues in the $\mathbf{C} 1$ domain and kinase domain common to all PKCs.

adjacent pseudosubstrate domain-mediated autoinhibitory intramolecular interactions that limits catalytic activity. This redox-dependent mechanism for PKC activation presumably underlies previous observations that $\mathrm{H}_{2} \mathrm{O}_{2}$ activates PKC without translocating the enzyme to membranes (Ohmori et al., 1998). In fact, $\mathrm{H}_{2} \mathrm{O}_{2}$ activates PKCs in association with a reverse redistribution of some PKC family members from the membrane to the cytosolic fraction in cardiomyocytes and certain other cell types (Goldberg et al., 1997; Rybin et al., 2004).

Oxidative modifications of cysteine residues in the C-terminal catalytic domain typically require considerably higher concentrations of $\mathrm{H}_{2} \mathrm{O}_{2}$ and result in enzyme inactivation. While the redox-sensitive residues in PKC kinase domains have never been localized, the redox-dependent mechanism that leads to inactivation of the catalytic subunit of protein kinase A (PKA) may be pertinent, given the high level of sequence homology between these enzymes. In the case of PKA, Cys ${ }^{199}$ in the activation loop is a target for $S$-glutathionylation (Humphries et al., 2002). While PKA contains a second redox sensitive cysteine at position 343 in the C-terminal variable region (that is not conserved in PKCs), there is evidence that oxidative modifications at $\mathrm{Cys}^{199}$ alone can be inactivating (Humphries et al., 2002, 2005). Two mechanisms have been identified. First, while Cys ${ }^{199}$ is not part of the catalytic mechanism, it is strategically located in in the $P+1$ loop that interacts with protein substrates. There is some evidence that oxidative modifications at this site reduce substrate-binding affinity. Second, Cys ${ }^{199}$ oxidation may inhibit enzyme activity through an indirect mechanism, by facilitating dephosphorylation of the neighboring $\mathrm{Thr}^{197}$ residue (Humphries et al., 2005). The PKA activation loop is phosphorylated at $\mathrm{Thr}^{197}$ during enzyme maturation. $\mathrm{Thr}^{197}$ phosphorylation is a stable 'priming' post-translational modification that is required to structure the catalytic pocket in an optimal conformation for catalysis. PKA-Thr ${ }^{197}$ phosphorylation is highly resistant to phosphatases under standard experimental conditions. However, Cys ${ }^{199}$ oxidation renders the adjacent activation loop $\mathrm{Thr}^{197}$ phosphorylation site susceptible to phosphatases; this underlies redox-dependent PKA inactivation. Of note, an activation loop sequence characterized by cysteine residue strategically located at the +2 position relative to the 'priming' threonine phosphorylation site that is required for optimal catalytic activity also is a characteristic feature of all $\mathrm{PKC}$ isoforms and many other AGC family kinases (Figure 3). Studies of PKC $\alpha$ suggest that this highly conserved chemical environment is 
functionally important, since the oxidative state promotes PKC $\alpha$ activation loop site dephosphorylation (Humphries et al., 2005). These results suggest that oxidative modifications within the activation loop may play a more general role to promote the dephosphorylation and inactivation of various PKCs.

\section{Tyrosine Phosphorylation of PKC $\delta$}

While translocation to membranes traditionally has been considered the hallmark of PKC activation (and traditionally has been used as a surrogate marker of enzyme activation in a cellular context), this allosteric model for PKC enzyme activation by lipid cofactors has two major limitations. First, a model that focuses exclusively on lipid cofactor-mediated translocation events that deliver the enzyme to membranes assumes that the cellular actions of PKCs are exclusively membrane-delimited. In fact, $\mathrm{PKC}$ isoforms are recovered in mitochondria and soluble fraction of cardiomyocytes subjected to various forms of oxidative stress (Goldberg et al., 1997; Konishi et al., 2001; Juhaszova et al., 2004); PKCs in this later fraction could underlie the wellrecognized actions of PKCs to regulate cardiac contractility by phosphorylating sarcomeric proteins in the contractile apparatus (at a site physically distant from the lipid bilayer). While there is some evidence that a subset of regulatory proteins associated with the contractile apparatus bind certain phospholipids (i.e., the actin capping protein CapZ interacts with $\mathrm{PIP}_{2}$ ) studies to date indicate that these lipid interactions play a structural role to regulate actin filament assemble (Russell et al., 2010). There is no evidence that sarcomeres are the site of phospholipid hydrolysis or the generation of DAG. Second, the canonical model for PKC activation views PKC's catalytic activity as an inherent property of the enzyme that is not altered by the activation process. This model does not adequately explain the diverse (and in some cases opposing) actions of certain PKC. For example, PKC $\delta$ has been implicated in both ischemia/reperfusion injury and cardioprotection (Mayr et al., 2004; Churchill et al., 2008).

Early studies showing that PKC $\delta$ is activated in a stimulusspecific manner in various cell types provided the rationale to consider a role for oxidative stress as an alternate mechanism to activate PKC $\delta$. Specifically, studies in cardiomyocytes established that GPCR agonists drive PKC $\delta$ to lipid-membranes membranes where it phosphorylates membrane-delimited substrates. The GPCR-dependent pathway does not lead to a detectable increase in PKC $\delta$ tyrosine phosphorylation. In contrast, oxidative stress releases PKC $\delta$ from membranes, activates Src, promotes PKC $\delta$-Src complex formation, and Src-dependent PKC $\delta$ tyrosine phosphorylation which increases PKC $\delta$ 's catalytic activity (Rybin et al., 2004). Several other Src family kinases (including Fyn, Lyn) that are expressed by cardiomyocytes can substitute for Src in the redox-dependet pathway that activates PKC $\delta$ (Rybin et al., 2004). Importantly, the redox-dependent increases in PKC $\delta$ tyrosine phosphorylation and PKC $\delta$ activity are detected in both the soluble and particulate subcellular compartments, suggesting that tyrosine phosphorylation confers lipid-independent catalytic activity.
PKC $\delta$ contains sites for tyrosine phosphorylation throughout the structure, including in the highly conserved regulatory and kinase domains and the intervening more flexible variable hinge region. Most of these tyrosine residues are unique to the PKC $\delta$ sequence; they are not conserved in other PKC family enzymes. Early studies from the Nishizuka laboratory implicated tyrosine phosphorylation as a mechanism to regulate $\mathrm{PKC} \delta$ catalytic activity, showing that the tyrosine-phosphorylated enzyme is a constitutively activate enzyme that no longer requires DAG as a cofactor to phosphorylate substrates (Konishi et al., 1997, 2001). It is worth noting that while the initial study from this group focused on phosphorylations at tyrosine residues in the regulatory domain $\left(\mathrm{Tyr}^{52}\right.$ and $\mathrm{Tyr}^{187}$ ) and a series of tyrosine residues in the kinase domain $\left[\mathrm{Tyr}^{451}, \mathrm{Tyr}^{469}\right.$, $\mathrm{Tyr}^{512}$, Tyr ${ }^{523}$ (Konishi et al., 1997)], the subsequent study concluded that a Src family kinase (in this case Lck) driven phosphorylation at $\mathrm{Tyr}^{311}$ (in the more variable flexible hinge region of the enzyme) mediates the $\mathrm{H}_{2} \mathrm{O}_{2}$-dependent increase in PKC $\delta$ activity (Konishi et al., 2001). Note: Tyr ${ }^{311}$ in rodent PKC $\delta$ corresponds to $\mathrm{Tyr}^{313}$ in human PKC $\delta$; the remainder of this review uses nomenclature based upon the rodent $\mathrm{PKC} \delta$ sequence. PKC $\delta$-Tyr ${ }^{311}$ phosphorylation was subsequently implicated in several other PKC $\delta$-dependent cellular responses (Lu et al., 2007; Nakashima et al., 2008). These results provided traction for the notion that a pathway that relies on tyrosine phosphorylation, rather than cysteine oxidation, underlies redox control of PKC $\delta$ activity.

Studies from our group combined molecular and biochemical approaches to implicate tyrosine phosphorylation as a key mechanism that mediates redox-dependent changes in PKC $\delta$ catalytic activity. In retrospect, our decision to use a rigorous in vitro kinase assay approach (which combined measurements of PKC $\delta$ autophosphorylation and PKC $\delta$-dependent phosphorylation of many sites on heterologous substrates) proved to be essential to expose a novel mechanism for the control of PKC $\delta$ activity. We used troponin complex as substrate in the in vitro kinase assays since this myofilament protein complex represents a physiologically important $\mathrm{PKC} \delta$ substrate in cardiomyocytes. Troponin complex is comprised of equimolar amounts of cardiac troponin I (cTnI), cardiac troponin $\mathrm{T}$ ( $\mathrm{cTnT}$ ), and cardiac troponin C. cTnI and cTnT (the "inhibitory" and 'tropomyosin-binding' subunits of the troponin complex) function to fine-tune calcium-dependent cardiac contractility. cTnI and cTnT both have several phosphorylation clusters that exert distinct effects on cardiac contractility. In an initial set of studies, we showed that PKC $\delta$ phosphorylates cTnI at $\mathrm{Ser}^{23} / \mathrm{Ser}^{24}$ when allosterically activated by lipid-cofactors; studies in skinned single cardiomyocytes linked this phosphorylation to an increase in the cross-bridge cycling rate and a reduction in myofilament calcium sensitivity (Sumandea et al., 2008). While this action of the allosterically activated PKC $\delta$ enzyme mimics the effects of the $\beta$-adrenergic receptor-PKA pathway, it is detected exclusively in our somewhat artificial reductionist model that is designed to expose mechanism; its physiologic relevance is doubtful, since the allosterically activated form of PKC $\delta$ does not localize the contractile machinery in cardiomyocytes. Rather, we identified a redox-induced Src-dependent change in PKC $\delta$ activity that is 
more physiologically relevant. We showed that $\mathrm{PKC} \delta$ acquires activity toward an additional site on cTnI (it phosphorylates cTnI at both $\mathrm{Ser}^{23} / \mathrm{Ser}^{24}$ and $\mathrm{Thr}^{144}$ ) and it also phosphorylates a number of threonine phosphorylation sites on cTnT during oxidative stress when the enzyme is $\mathrm{Tyr}^{311}$-phosphorylated by Src. This change in PKC $\delta$ 's enzymology mediates the redoxdependent decrease in maximum tension and cross-bridge kinetics (i.e., a functionally distinct and physiologically relevant mechanism for $\operatorname{PKC} \delta$ regulation of contractility (Sumandea et al., 2008)). The additional observation that the Src-dependent acquisition of cTnI-Thr ${ }^{144}$ and cTnT-Thr kinase activity is completely abrogated by a Y311F substitution implicated $\mathrm{Tyr}^{311}$-phosphorylation as the mechanism underlying the Src-dependent increase in PKC $\delta$ activity (Sumandea et al., 2008).

A mechanism that might explain how a phosphorylation at $\mathrm{Tyr}^{311}$ (in the hinge region, outside the catalytic core of the enzyme) might lead to a change in PKC $\delta$ 's enzymology is not obvious. However, we focused on the intriguing observation that $\mathrm{PKC} \delta$ contains a phosphotyrosine binding $\mathrm{C} 2$ domain with a consensus binding sequence [(Y/F)-(S/A)-(V/I)-pY$(\mathrm{Q} / \mathrm{R})-\mathrm{X}-(\mathrm{Y} / \mathrm{F})]$ that resembles the sequence flanking $\mathrm{Tyr}^{311}$ (Benes et al., 2005). Tyr ${ }^{311}$ is the only phosphorylation site in $\mathrm{PKC} \delta$ flanked by residues that conform to a $\mathrm{PKC} \delta-\mathrm{C} 2$ domain consensus-binding motif (VGI-Y $311-Q G F)$. We used a FRET-based approach to show that PKC $\delta$ 's phosphotyrosinebinding $\mathrm{C} 2$ domain interacts with the $\mathrm{Tyr}^{311}$-phosphorylated V3 region (Figure 4; Gong et al., 2015). We then showed that this interaction controls $\mathrm{PKC} \delta$ catalytic activity indirectly, by regulating $\mathrm{PKC} \delta$ phosphorylation at a novel regulatory phosphorylation site at $\operatorname{Ser}^{357}$ (rodent sequence, corresponding to $\operatorname{Ser}^{359}$ in human PKC $\delta$ ). Ser ${ }^{357}$ sits at the tip of the Glyrich ATP-positioning loop (G-loop) within the small lobe of the kinase domain. The G-loop sequence is highly conserved in most protein kinases and it forms a flexible clamp that orients the $\gamma$-phosphate of ATP for transfer to substrate; localized changes in the position of this loop influence the kinetics of nucleotide binding and catalysis. We showed that PKC $\delta$ displays a high level of $\mathrm{S}^{357}$ phosphorylation in resting cells and that the redox-induced docking interaction between the $\mathrm{C} 2$ domain and the $\mathrm{Tyr}^{311}$-phosphorylated hinge region leads to $\mathrm{S}^{357}$ dephosphorylation.

Mutagenesis studies implicated G-loop dephosphorylation as a mechanism to regulate two major aspects of $\mathrm{PKC} \delta$ 's enzymology (Gong et al., 2015). First, we showed that the PKC $\delta$-S357A mutant is a constitutively active lipid-independent enzyme. The observation that a single non-phosphorylatable S357A substitution in the G loop confers a high level of lipidindependent catalytic activity provides a mechanism to account for the cellular actions of PKC $\delta$ in cells subjected to oxidative stress, where PKC $\delta$ phosphorylates substrates throughout the cell (not just on lipid membranes).

Second, we showed that the S357A substitution changes the enzyme's substrate specificity (Gong et al., 2015). In way of background, most studies that define enzyme-substrate specificity focus on consensus phosphorylation motifs, residues that are either preferred or deselected at positions flanking the phosphoacceptor-site (or P-site). The literature has generally overlooked the fact that PKC isoforms (and PKA - enzymes typically characterized as Ser/Thr kinases) show a clear preference for substrates with a P-site Ser

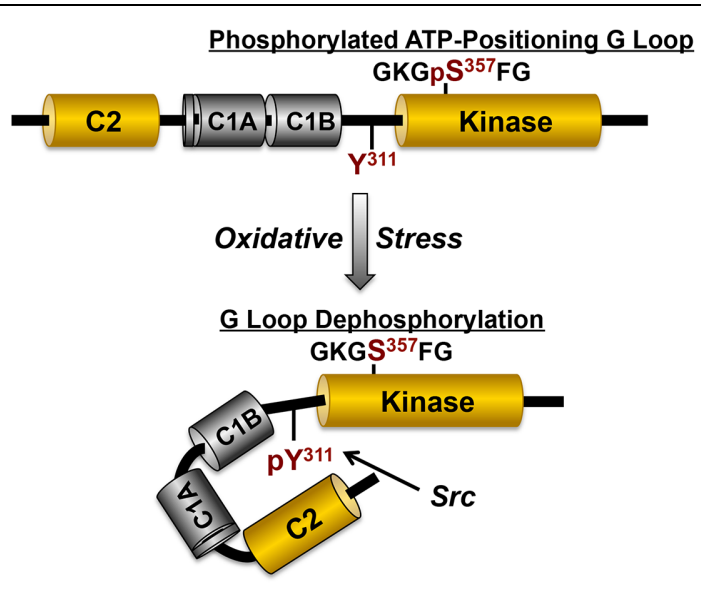

* Src phosphorylates PKC트 at $Y^{311}$.

* This generates a docking site for the $\mathrm{C2}$ domain.

* C2 domain docking to the $\mathrm{Y}^{311}$-phosphorylated hinge region leads to $\mathrm{G}$ loop dephosphorylation at $\mathbf{S}^{357}$.

* G loop dephosphorylation underlies the redox-dependent increase in PKC $\delta$ activity

FIGURE 4 | The redox-dependent pathway involving Src that switches PKC $\delta$ from a lipid-dependent Ser kinase to a lipid independent Ser/Thr kinase. The structural domains that mediate redox-dependent activation of
PKC $\delta$ are highlighted in yellow, with the specific phosphorylation sites in the variable hinge region $\left(\mathrm{Tyr}^{311}\right)$ and kinase domain $\left(\mathrm{Ser}^{357}\right)$ that are key for redox-dependent activation in red (see text). 
residue. In fact, our early studies showed that the allosterically activated PKC $\delta$ enzyme phosphorylates cTnI at $\mathrm{Ser}^{23} / \mathrm{Ser}^{24}$, but it does not phosphorylate $\mathrm{Thr}$ phosphorylation sites on $\mathrm{cTnI}$ or $\mathrm{cTnT}$. The observation that the redox-activated $\left(\mathrm{Tyr}^{311}\right.$-phosphorylated/Ser ${ }^{357}$ dephosphorylated) form of PKC $\delta$ acquires activity toward threonine phosphorylation sites on cTnI and cTnT provided the rationale to test whether G loop phosphorylation constitutes a mechanism to regulate PKC 8 's P-site specificity. By using a series of rigorous biochemical approaches, we were able to show that the G loop phosphorylation site - which (according to structural studies of the related PKA catalytic subunit) occupies a tight space adjacent to the P-site on substrates - regulates P-site specificity. We showed that a non-phosphorylatable S357A substitution facilitates (and a phosphomimetic S357E substitution completely abrogates) PKC $\delta$ 's Thr kinase activity. Activity toward substrates with Ser-phosphoacceptor sites is not affected. These results implicate G loop Ser ${ }^{357}$ phosphorylation as a novel dynamically regulated mechanism that controls PKC $\delta$ 's P-site specificity. It is worth emphasizing that a post-translational modification within the kinase domain that calibrates an enzyme's P-site specificity in a stimulus-specific manner is unprecedented. It is tempting to speculate that the stimulus-specific differences in PKC $\delta$ phosphorylation profiles (that impact on substrate specificity) underlie PKC $\delta$ 's diverse roles in both the pathogenesis of ischemia-reperfusion injury and as a mediator of ischemic preconditioning (Steinberg, 2012). As a technical note, it also is worth noting that our studies expose an important and underappreciated caveat related to previous studies that have characterized PKC $\delta$ enzyme activity. Our studies emphasize that measurements of stimulus-dependent changes in PKC $\delta$ kinase activity are likely to differ, depending on the readout used in the assay - and particularly, the nature of the substrate (whether the substrate P-site is a Ser or Thr residue). This may explain some of the confounding inconsistencies in previous published literature.

\section{References}

Adwan, T. S., Ohm, A. M., Jones, D. N., Humphries, M. J., and Reyland, M. E. (2011). Regulated binding of importin- $\alpha$ to protein kinase C- $\delta$ in response to apoptotic signals facilitates nuclear import. J. Biol. Chem. 286, 35716-35724. doi: 10.1074/jbc.M111.255950

Andersson, D. C., Betzenhauser, M. J., Reiken, S., Meli, A. C., Umanskaya, A., Xie, W., et al. (2011). Ryanodine receptor oxidation causes intracellular calcium leak and muscle weakness in aging. Cell Metab. 14, 196-207. doi: 10.1016/j.cmet.2011.05.014

Benes, C. H., Wu, N., Elia, A. E., Dharia, T., Cantley, L. C., and Soltoff, S. P. (2005). The C2 domain of PKC $\delta$ is a phosphotyrosine binding domain. Cell 121, 271-280. doi: 10.1016/j.cell.2005.02.019

Churchill, E., Budas, G., Vallentin, A., Koyanagi, T., and MochlyRosen, D. (2008). PKC isozymes in chronic cardiac disease: possible therapeutic targets? Annu. Rev. Pharmacol. Toxicol. 48, 569-599. doi: 10.1146/annurev.pharmtox.48.121806.154902

Goldberg, M., Zhang, H. L., and Steinberg, S. F. (1997). Hypoxia alters the subcellular distribution of protein kinase $\mathrm{C}$ isoforms in neonatal rat ventricular myocytes. J. Clin. Invest. 99, 55-61. doi: 10.1172/JCI1 19133

Gong, J., Yao, Y., Zhang, P., Udayasuryan, B., Komissarova, E. V., Chen, J., et al. (2015). The C2 domain and altered ATP-binding loop phosphorylation at $\operatorname{Ser}^{359}$
Finally, it is worth mentioning that other tyrosine residues in the regulatory region of $\mathrm{PKC} \delta$, that figure prominently in the transduction of genotoxic stress responses, can also become phosphorylated in the setting of oxidative stress (Adwan et al., 2011). For example, PKC $\delta$ phosphorylation at $\mathrm{Tyr}^{64}$ and $\mathrm{Tyr}^{155}$ (sites in the regulatory domain) leads to a conformational change that exposes bipartite nuclear localization signal in the catalytic domain. This results in an interaction with importin- $\alpha$, which is key for the nuclear translocation of full-length PKC $\delta$ and various PKC $\delta$-driven proapoptotic responses. Oxidative stress also undoubtedly increases PKC $\delta$ phosphorylation at other tyrosine residues throughout the enzyme, but the controls and consequences of other redoxinduced tyrosine phosphorylations (either in a cardiomyocyte context or with respect to their effects on catalytic activity) have not been interrogated. This remains fertile ground for future investigations.

\section{Concluding Remarks}

This review summarizes our current understanding of various redox-activated mechanisms that regulate signaling by PKCs. While this narrative considers individual mechanisms separately, PKCs are subject to an elaborate and complex assortment of redox-driven modifications in various pathophysiologic settings. An understanding of the ensemble effects of these redox-driven events presents both a challenge and an opportunity for the development of novel PKC-targeted therapeutics.

\section{Acknowledgment}

This work was supported by HL77860 and HL123061.

mediate the redox-dependent increase in protein kinase C- $\delta$ activity. Mol. Cell. Biol. 35, 1727-1740. doi: 10.1128/MCB.01436-14

Guo, J., Gertsberg, Z., Ozgen, N., and Steinberg, S. F. (2009). p66Shc links alpha1adrenergic receptors to a reactive oxygen species-dependent AKT-FOXO3A phosphorylation pathway in cardiomyocytes. Circ. Res. 104, 660-669. doi: 10.1161/CIRCRESAHA.108.186288

Hirotani, S., Otsu, K., Nishida, K., Higuchi, Y., Morita, T., Nakayama, H., et al. (2002). Involvement of nuclear factor-kappaB and apoptosis signal-regulating kinase 1 in G-protein-coupled receptor agonist-induced cardiomyocyte hypertrophy. Circulation 105, 509-515. doi: 10.1161/hc0402. 102863

Humphries, K. M., Deal, M. S., and Taylor, S. S. (2005). Enhanced dephosphorylation of cAMP-dependent protein kinase by oxidation and thiol modification. J. Biol. Chem. 280, 2750-2758. doi: 10.1074/jbc.M410242200

Humphries, K. M., Juliano, C., and Taylor, S. S. (2002). Regulation of cAMPdependent protein kinase activity by glutathionylation. J. Biol. Chem. 277, 43505-43511. doi: 10.1074/jbc.M207088200

Juhaszova, M., Zorov, D. B., Kim, S. H., Pepe, S., Fu, Q., Fishbein, K. W., et al. (2004). Glycogen synthase kinase- $\beta$ mediates convergence of protection signaling to inhibit the mitochondrial permeability transition pore. J. Clin. Invest. 113, 1535-1549. doi: 10.1172/JCI19906

Kambayashi, Y., Takekoshi, S., Tanino, Y., Watanabe, K., Nakano, M., Hitomi, Y., et al. (2007). Various molecular species of diacylglycerol hydroperoxide activate 
human neutrophils via PKC activation. J. Clin. Biochem. Nutr. 41, 68-75. doi: 10.3164/jcbn.2007009

Kang, M. Y., Zhang, Y., Matkovich, S. J., Diwan, A., Chishti, A. H., and Dorn, G. W. (2010). Receptor-independent cardiac protein kinase $\mathrm{C}-\alpha$ activation by calpain-mediated truncation of regulatory domains. Circ. Res. 107, 903-912. doi: 10.1161/CIRCRESAHA.110.220772

Kishimoto, A., Mikawa, K., Hashimoto, K., Yasuda, I., Tanaka, S., Tominaga, M., et al. (1989). Limited proteolysis of protein kinase C subspecies by calciumdependent neutral protease (calpain). J. Biol. Chem. 264, 4088-4092.

Knapp, L. T., and Klann, E. (2000). Superoxide-induced stimulation of protein kinase $\mathrm{C}$ via thiol modification and modulation of zinc content. J. Biol. Chem. 275, 24136-24145. doi: 10.1074/jbc.M002043200

Knock, G. A., and Ward, J. P. (2011). Redox regulation of protein kinases as a modulator of vascular function. Antioxid. Redox Signal. 15, 1531-1547. doi: 10.1089 /ars.2010.3614

Konishi, H., Tanaka, M., Takemura, Y., Matsuzaki, H., Ono, Y., Kikkawa, U., et al. (1997). Activation of protein kinase C by tyrosine phosphorylation in response to H2O2. Proc. Natl. Acad. Sci. U.S.A. 94, 11233-11237. doi: 10.1073/pnas.94.21.11233

Konishi, H., Yamauchi, E., Taniguchi, H., Yamamoto, T., Matsuzaki, H., Takemura, Y., et al. (2001). Phosphorylation sites of protein kinase $\mathrm{C} \delta$ in $\mathrm{H} 2 \mathrm{O} 2$ treated cells and its activation by tyrosine kinase in vitro. Proc. Natl. Acad. Sci. U.S.A. 98, 6587-6592. doi: 10.1073/pnas.111158798

Korichneva, I., Hoyos, B., Chua, R., Levi, E., and Hammerling, U. (2002). Zinc release from protein kinase $\mathrm{C}$ as the common event during activation by lipid second messenger or reactive oxygen. J. Biol. Chem. 277, 44327-44331. doi: 10.1074/jbc.M205634200

Kuster, G. M., Pimentel, D. R., Adachi, T., Ido, Y., Brenner, D. A., Cohen, R. A., et al. (2005). Alpha-adrenergic receptor-stimulated hypertrophy in adult rat ventricular myocytes is mediated via thioredoxin-1-sensitive oxidative modification of thiols on Ras. Circulation 111, 1192-1198. doi: 10.1161/01.CIR.0000157148.59308.F5

Lu, W., Finnis, S., Xiang, C., Lee, H. K., Markowitz, Y., Okhrimenko, H., et al. (2007). Tyrosine311 is phosphorylated by c-Abl and promotes the apoptotic effect of PKC $\delta$ in glioma cells. Biochem. Biophys. Res. Commun. 352, 431-436. doi: 10.1016/j.bbrc.2006.11.028

MacKay, C. E., and Knock, G. A. (2014). Control of vascular smooth muscle function by Src-family kinases and reactive oxygen species in health and disease. J. Physiol. doi: 10.1113/jphysiol.2014.285304 [Epub ahead of print].

Mayr, M., Metzler, B., Chung, Y. L., Mcgregor, E., Mayr, U., Troy, H., et al. (2004). Ischemic preconditioning exaggerates cardiac damage in PKC $\delta$ null mice. Am. J. Physiol. Heart Circ. Physiol. 287, H946-H956. doi: 10.1152/ajpheart.00878.2003

Nakamura, K., Fushimi, K., Kouchi, H., Mihara, K., Miyazaki, M., Ohe, T., et al. (1998). Inhibitory effects of antioxidants on neonatal rat cardiac myocyte hypertrophy induced by tumor necrosis factor-alpha and angiotensin II. Circulation 98, 794-799. doi: 10.1161/01.CIR.98.8.794

Nakashima, H., Frank, G. D., Shirai, H., Hinoki, A., Higuchi, S., Ohtsu, H., et al. (2008). Novel role of protein kinase C- $\delta$ Tyr311 phosphorylation in vascular smooth muscle cell hypertrophy by angiotensin II. Hypertension 51, 232-238. doi: 10.1161/HYPERTENSIONAHA.107.101253

Ohmori, S., Shirai, Y., Sakai, N., Fujii, M., Konishi, H., Kikkawa, U., et al. (1998). Three distinct mechanisms for translocation and activation of the delta subspecies of protein kinase C. Mol. Cell. Biol. 18, 5263-5271.

Paulsen, C. E., Truong, T. H., Garcia, F. J., Homann, A., Gupta, V., Leonard, S. E., et al. (2012). Peroxide-dependent sulfenylation of the EGFR catalytic site enhances kinase activity. Nat. Chem. Biol. 8, 57-64. doi: 10.1038/ nchembio.736

Roussel, J., Thireau, J., Brenner, C., Saint, N., Scheuermann, V., Lacampagne, A., et al. (2015). Palmitoyl-carnitine increases RyR2 oxidation and sarcoplasmic reticulum $\mathrm{Ca} 2+$ leak in cardiomyocytes: role of adenine nucleotide translocase. Biochim. Biophys. Acta 1852, 749-758. doi: 10.1016/j.bbadis.2015.01.011

Russell, B., Curtis, M. W., Koshman, Y. E., and Samarel, A. M. (2010). Mechanical stress-induced sarcomere assembly for cardiac muscle growth in length and width. J. Mol. Cell. Cardiol. 48, 817-823. doi: 10.1016/j.yjmcc.2010.02.016

Rybin, V. O., Guo, J., Sabri, A., Elouardighi, H., Schaefer, E., and Steinberg, S. F. (2004). Stimulus-specific differences in protein kinase $C-\delta$ localization and activation mechanisms in cardiomyocytes. J. Biol. Chem. 279, 19350-19361. doi: 10.1074/jbc.M311096200
Santos, C. X., Anilkumar, N., Zhang, M., Brewer, A. C., and Shah, A. M. (2011). Redox signaling in cardiac myocytes. Free Radic. Biol. Med. 50, 777-793. doi: 10.1016/j.freeradbiomed.2011.01.003

Santulli, G., Pagano, G., Sardu, C., Xie, W., Reiken, S., D’ascia, S. L., et al. (2015). Calcium release channel RyR2 regulates insulin release and glucose homeostasis. J. Clin. Invest. 125, 1968-1978. doi: 10.1172/JCI79273

Stahelin, R. V., Kong, K. F., Raha, S., Tian, W., Melowic, H. R., Ward, K. E., et al. (2012). Protein kinase C- $\theta$ C2 domain is a phosphotyrosine binding module that plays a key role in its activation. J. Biol. Chem. 287, 30518-30528. doi: $10.1074 /$ jbc.M112.391557

Steinberg, S. F. (2008). Structural basis of protein kinase C isoform function. Physiol. Rev. 88, 1341-1378. doi: 10.1152/physrev.00034.2007

Steinberg, S. F. (2012). Cardiac actions of protein kinase C isoforms. Physiology (Bethesda) 27, 130-139. doi: 10.1152/physiol.00009.2012

Sumandea, M. P., Rybin, V. O., Hinken, A. C., Wang, C., Kobayashi, T., Harleton, E., et al. (2008). Tyrosine phosphorylation modifies PKC $\delta$-dependent phosphorylation of cardiac troponin I. J. Biol. Chem. 283, 22680-22689. doi: 10.1074/jbc.M802396200

Tocchetti, C. G., Molinaro, M., Angelone, T., Lionetti, V., Madonna, R., Mangiacapra, F., et al. (2015). Nitroso-Redox Balance and Modulation of Basal Myocardial Function: an Update from the Italian Society of Cardiovascular Research (SIRC). Curr. Drug Targets doi: 10.2174/1389450116666150304103517 [Epub ahead of print].

Truong, T. H., and Carroll, K. S. (2012). Redox regulation of epidermal growth factor receptor signaling through cysteine oxidation. Biochemistry 51, 99549965. doi: 10.1021/bi301441e

Umanskaya, A., Santulli, G., Xie, W., Andersson, D. C., Reiken, S. R., and Marks, A. R. (2014). Genetically enhancing mitochondrial antioxidant activity improves muscle function in aging. Proc. Natl. Acad. Sci. U.S.A. 111, 1525015255. doi: 10.1073/pnas.1412754111

Wang, X., Mccullough, K. D., Franke, T. F., and Holbrook, N. J. (2000). Epidermal growth factor receptor-dependent Akt activation by oxidative stress enhances cell survival. J. Biol. Chem. 275, 14624-14631. doi: 10.1074/jbc.275.19.14624

Wang, X. T., Mccullough, K. D., Wang, X. J., Carpenter, G., and Holbrook, N. J. (2001). Oxidative stress-induced phospholipase C-gamma 1 activation enhances cell survival. J. Biol. Chem. 276, 28364-28371. doi: 10.1074/jbc.M102693200

Xu, W., Doshi, A., Lei, M., Eck, M. J., and Harrison, S. C. (1999). Crystal structures of c-Src reveal features of its autoinhibitory mechanism. Mol. Cell 3, 629-638. doi: 10.1016/S1097-2765(00)80356-1

Yamakawa, H., Banno, Y., Nakashima, S., Yoshimura, S., Sawada, M., Nishimura, Y., et al. (2001). Crucial role of calpain in hypoxic PC12 cell death: calpain, but not caspases, mediates degradation of cytoskeletal proteins and protein kinase $\mathrm{C}-\alpha$ and $-\delta$. Neurol. Res. 23, 522-530. doi: $10.1179 / 016164101101198776$

Zalk, R., Lehnart, S. E., and Marks, A. R. (2007). Modulation of the ryanodine receptor and intracellular calcium. Annu. Rev. Biochem. 76, 367-385. doi: 10.1146/annurev.biochem.76.053105.094237

Zhang, H., Davies, K. J., and Forman, H. J. (2015). TGFbetal rapidly activates Src through a non-canonical redox signaling mechanism. Arch. Biochem. Biophys. 568, 1-7. doi: 10.1016/j.abb.2015.01.001

Zhang, Y., Matkovich, S. J., Duan, X., Diwan, A., Kang, M. Y., and Dorn, G. W. II. (2011). Receptor-independent protein kinase C- $\alpha$ (PKC $\alpha)$ signaling by calpain-generated free catalytic domains induces HDAC5 nuclear export and regulates cardiac transcription. J. Biol. Chem. 286, 26943-26951. doi: 10.1074/jbc.M111.234757

Conflict of Interest Statement: The author declares that the research was conducted in the absence of any commercial or financial relationships that could be construed as a potential conflict of interest.

Copyright (c) 2015 Steinberg. This is an open-access article distributed under the terms of the Creative Commons Attribution License (CC BY). The use, distribution or reproduction in other forums is permitted, provided the original author(s) or licensor are credited and that the original publication in this journal is cited, in accordance with accepted academic practice. No use, distribution or reproduction is permitted which does not comply with these terms. 